Research Paper

\title{
The latest exploration of staging and prognostic classification for pancreatic neuroendocrine tumors: a large population-based study
}

\author{
Shanshan $\mathrm{Gao}^{1,2^{*}}$, Ning $\mathrm{Pu}^{3^{*}}$, Lingxiao Liu ${ }^{1,2}$, Changyu $\mathrm{Li}^{1,2}$, Xuefeng $\mathrm{Xu}^{3}$, Xiaolin Wang ${ }^{1,2} \bowtie$, Wenhui Lou ${ }^{3 凶}$ \\ 1. Shanghai Institute of Medical Imaging, Shanghai, 200032, People's Republic of China \\ 2. Department of Interventional Radiology, Zhongshan Hospital, Fudan University, Shanghai, 200032, People's Republic of China \\ 3. Department of General Surgery, Zhongshan Hospital, Fudan University, Shanghai, 200032, People's Republic of China \\ *These authors contributed equally to this work \\ $\square$ Corresponding authors: Wenhui Lou, Department of General Surgery, Zhongshan Hospital, Fudan University, 180 Fenglin Road, Shanghai, 200032, P.R. \\ China. E-mail: lou.wenhui@zs-hospital.sh.cn; Xiaolin Wang, Department of Interventional Radiology, Zhongshan Hospital, Fudan University, 180 Fenglin Road, \\ Shanghai, 200032, P.R. China. E-mail: wang.xiaolin@zs-hospital.sh.cn \\ ( ) Ivyspring International Publisher. This is an open access article distributed under the terms of the Creative Commons Attribution (CC BY-NC) license \\ (https://creativecommons.org/licenses/by-nc/4.0/). See http://ivyspring.com/terms for full terms and conditions.
}

Received: 2017.12.24; Accepted: 2018.01.27; Published: 2018.04.19

\begin{abstract}
Background: A modified European Neuroendocrine Tumor Society (mENETS) staging system has been confirmed to be more suitable for pancreatic neuroendocrine tumors (pNETs) when compared to the American Joint Committee on Cancer (AJCC) or the European Neuroendocrine Tumor Society (ENETS) systems in the last few years. However, the importance of $\mathrm{N}$ stage has been recently published with several significant updates.

Methods: SEER registry $(n=2,209)$ was used to evaluate the application of the AJCC 7th staging system, ENETS staging system, mENETS staging system and reformed ENETS (rENETS) staging system in this study.

Results: For the ENETS staging system, patients with stage I disease had a similar prognosis to patients with stage II disease $(P=0.154)$, and patients with stage IIIA and stage IIIB diseases showed adverse prognostic potential. The proportion of patients with stage III diseases using AJCC 7th staging system was extremely lower than those with mENETS staging system or rENETS staging system $(3.6 \%, 23.0 \%$ and $23.7 \%$, respectively). Furthermore, the hazard ratio of death for patients with stage II or III disease using rENETS staging system was slightly higher than that of mENETS staging system. Besides, survival curves were better separated by rENETS staging system. A prognostic nomogram for overall survival (OS) was formulated to obtain superior discriminatory abilities.

Conclusions: The rENETS staging system has superior distribution in proportion than the AJCC 7th, ENETS or mENETS staging system, and one more accurate and advanced predictive model will be achieved via the incorporation to be adopted in clinical practice.
\end{abstract}

Key words: rENETS, mENETS, pancreatic neuroendocrine tumor, nomogram, decision curve analysis

\section{Introduction}

Of all pancreatic tumors, pancreatic neuroendocrine tumors (pNETs) only account for $1 \%$ to $5 \%$ and are rare and highly heterogeneous neuroendocrine tumors ${ }^{1-3}$. With the attention and technology improvement, the incidence has increased in 2 to 3 fold over the past decades ${ }^{4-6}$. Due to its peculiarity of occult malignancy and indolent characteristics, pNETs patients have a limited survival outcome with a 5-year survival rate of $80 \%-90 \%$ after resected surgery $3,7,8$. Thus, a powerful and accurate staging 
system applied for survival prediction and risk stratification is urgently required $7,9,10$.

A consensus published for TNM staging classification of pNETs by the European Neuroendocrine Tumor Society (ENETS) in 2006 has been validated and applied in amounts of studies ${ }^{11-15}$. Apart from that, various studies have also demonstrated the applicability of the AJCC 7th staging system for pNETs 16-19. Considering their drawbacks, Guopei Luo et $\mathrm{al}^{7}$ creatively put forward the modified ENETS (mENETS) staging classification which maintained the ENETS T, N and M definitions and also adopted the AJCC 7th staging definitions. The mENETS staging system overcame the disorder both between the stage I and IIA diseases and between stage IIIA and IIIB diseases using the ENETS staging system, and the small number of stage III diseases using the AJCC 7th staging system. However, the mENETS stage system was not applicable for all situations and further improvement was called for to address the problems that clinical practitioners may encounter. As the AJCC 8th staging system for pancreatic ductal adenocarcinoma (PDAC) allowed for finer risk stratification of patients with resected tumors according to extent of nodal involvement ${ }^{20}$, the classification changes to the AJCC staging system for PDAC may also benefit for the improvement of ENETS staging system.

This study was mainly aimed to obtain a more accurate staging system in a framework of analysis of the application based on ENETS and AJCC 8th N classifications for PDAC. The reformed ENETS (rENETS) staging classification would maintain the ENETS T, M definitions and AJCC 8th $\mathrm{N}$ definitions of PDAC and adopt the AJCC 8th staging definitions of PDAC. Combined with the created rENETS staging system, we managed to formulate a novel nomogram to improve the discrimination ability of survival prediction.

\section{Materials and methods}

\section{Patient population and data source}

The data used in our study were retrieved from the Surveillance, Epidemiology, and End Results (SEER) database registry of National Cancer Institute. The pNET patients were enrolled based on column of Site and Morphology (Primary Site - labeled) for tumor of pancreas which was labeled as C25.0 to C25.9. Then according to the International Classification of Diseases for Oncology (3rd edition) for tumor of histology / behavior, pancreatic endocrine tumor, malignant $(8150 / 3)$, insulinoma, malignant (8151/3), glucagonoma, malignant (8152/3), gastrinoma, malignant $(8153 / 3)$, mixed pancreatic endocrine and exocrine tumor, malignant (8154/3), vipoma, malignant (8155/3), carcinoid tumor $(8240 / 2)$, Enterochromaffin cell carcinoid $(8241 / 3)$, enterochromaffin-like cell tumor, malignant $(8242 / 3)$, goblet cell carcinoid (8243/3), mixed adenoneuroendocrine carcinoma (8244/3), adenocarcinoid tumor $(8245 / 3)$, neuroendocrine carcinoma (8246), and atypical carcinoid tumor $(8249 / 3)$ were all included. Another selection criterion was the diagnosis of year from 2004 to 2012.

The following data were obtained for each patients: sex, race, age, primary site, primary lesion amounts, tumor type, grade, surgery decision, TNM stage and survival information. The TNM stage of AJCC 7th edition, ENETS, mENETS and rENETS were evaluated according to the following codes: collaborative stage (CS) tumor size 2004, CS extension 2004, CS lymph nodes 2004, CS metastases at DX 2004, derived AJCC stage group (6th edition; 2004+), derived AJCC stage group (7th edition; 2010+). In addition, patients lacking of survival information or certain TNM stage, or with unknown characteristics were excluded in this study.

\section{Statistical analysis}

Statistical analyses were conducted using $R$ project version 3.3.3 (http:/ / www.r-project.org/) and SPSS 21.0 statistical package (SPSS Inc., Chicago, IL, USA) for Windows. The overall survival (OS) depicted by Graphpad Prism6 program was analyzed using Kaplan-Meier curves and compared by the log-rank test. The Cox proportional hazards regression model was used for univariate and multivariate analyses and hazard ratios (HR) to identify independent prognostic factors, and $P$ value less than 0.05 was defined statistically significant.

One novel prognostic nomogram for OS based on the results of multivariate analysis was established by the rms package in $\mathrm{R}$ project. The predictive performance of the nomogram was further measured by concordance index (C-index), calibration curve and decision curve analysis (DCA) ${ }^{21}$. Bootstraps with 1,200 resample were used for these activities. The predictive comparisons were evaluated by C-index between the established nomogram and other independent index.

\section{Results}

\section{Clinicopathological characteristics}

According to the whole criteria above, 2,209 patients with histopathological confirmed pNETs from the SEER database were finally enrolled in this study and the detailed clinicopathological characteristics were tabulated in Table 1 . In this whole population, there were 1182 males and 1027 females, with a median age of 60 (ranging from 18 to 91). 
Approximately $80 \%$ of patients were Caucasian. The number of patients with a tumor located at the head of pancreas was similar to that at the tail of pancreas. pNETs with only one primary lesion constituted the majority $(77.4 \%)$ and $83.2 \%$ of patients were diagnosed as nonfunctional pNETs. Approximately $85 \%$ of patients possessed high and intermediate pathological differentiation. In the decision of therapy, 1557 patients received surgery and 599 patients were not recommended for surgery, while 53 patients were recommended for surgery but somehow not performed. The median survival time was 98.0 months (ranges, 0-131 months). Furthermore, the OS rates at 5, 8 and 10 years were $62.5 \%, 50.3 \%$ and $43.1 \%$ respectively. According to the AJCC 7th edition, ENETS stage, mENETS stage and rENETS stage, the number of patients with separate stage was recorded respectively.

\section{Independent prognostic factors}

The univariate analysis results showed that age, sex, tumor type, grade, surgery and all TNM stages could be considered as influence factors in pNETS survival (Table 1). In the further multivariate analysis for OS, age, sex, grade, surgery and all TNM stages remained as independent risk indicators for tumor OS (Table 2).

\section{AJCC 7th staging system and survival}

Only 3.6\% (79 of 2,209) of patients could be classified into stage III tumors on the basis of the AJCC 7th staging classification (Table 1), and stage IIA and stage IIB tumors showed the adverse prognostic trends (Fig. 1a). In addition, the discrimination between stage III and IV diseases was smaller in the first half of survival curve, while it showed similarity between stage II and III diseases in the second half (Fig. 1b). The HR of stage III by multivariable analyses was 2.236, which was comparable to stage IV with $\mathrm{HR}$ as 3.194 when compared with stage I tumor (Table 2).

\section{ENETS staging system and survival}

It was awful that all survival curves except stage IV disease were complex and disordered. The overlaps were noticed between the ENETS classification of stage I, IIA and IIB disease, and between stage IIIA and IIIB disease which was even upside down (Fig. 1c). Furthermore, it was obvious that stage I and II disease couldn't be distinguished from each other (Fig. 1d) and no statistical significance was analyzed with HR by a multivariable analysis between stage I and II disease (HR, 1.338; P $=0.154 ;$ Table 2 ).
Table 1. Clinicopathologic characteristics: univariate analysis.

\begin{tabular}{|c|c|c|c|c|}
\hline \multirow[t]{2}{*}{ Variables } & \multicolumn{2}{|c|}{ Patients $\mathrm{N}=\mathbf{2 2 0 9}$} & \multicolumn{2}{|l|}{ OS } \\
\hline & No. & $\%$ & $P$ value & HR $(95 \%$ CI) \\
\hline Age, years & & & $<0.001$ & $1.901(1.721-2.100$ \\
\hline$<40$ & 180 & 8.1 & & \\
\hline $40-59$ & 886 & 40.1 & & \\
\hline $60-79$ & 994 & 45.0 & & \\
\hline$\geq 80$ & 149 & 6.7 & & \\
\hline Sex & & & $<0.001$ & $1.343(1.168-1.546)$ \\
\hline Female & 1027 & 46.5 & & \\
\hline Male & 1182 & 53.5 & & \\
\hline Race & & & 0.394 & $1.051(0.937-1.179)$ \\
\hline Asian & 183 & 8.3 & & \\
\hline Black & 247 & 11.2 & & \\
\hline White & 1762 & 79.8 & & \\
\hline Others & 17 & 0.8 & & \\
\hline Primary site & & & 0.201 & $0.961(0.905-1.021)$ \\
\hline Head & 668 & 30.2 & & \\
\hline Body & 289 & 13.1 & & \\
\hline Tail & 727 & 32.9 & & \\
\hline Others & 525 & 23.8 & & \\
\hline Primary lesion amounts & & & 0.281 & $1.069(0.947-1.206)$ \\
\hline 1 & 1709 & 77.4 & & \\
\hline 2 & 388 & 17.6 & & \\
\hline$\geq 3$ & 112 & 5.1 & & \\
\hline Tumor type & & & $<0.001$ & $1.501(1.211-1.859$ \\
\hline Functional & 371 & 16.8 & & \\
\hline Nonfunctional & 1838 & 83.2 & & \\
\hline Grade & & & $<0.001$ & $2.473(2.278-2.685)$ \\
\hline High & 1449 & 65.6 & & \\
\hline Intermediate & 399 & 18.1 & & \\
\hline Low & 361 & 16.3 & & \\
\hline Surgery & & & $<0.001$ & $2.360(2.198-2.533)$ \\
\hline Performed & 1557 & 70.5 & & \\
\hline $\begin{array}{l}\text { Recommended but not } \\
\text { performed }\end{array}$ & 53 & 2.4 & & \\
\hline Not recommended & 599 & 27.1 & & \\
\hline AJCC 7th stage & & & $<0.001$ & $1.532(1.465-1.601)$ \\
\hline IA & 378 & 17.1 & & \\
\hline IB & 409 & 18.5 & & \\
\hline IIA & 195 & 8.8 & & \\
\hline IIB & 391 & 17.7 & & \\
\hline III & 79 & 3.6 & & \\
\hline IV & 757 & 34.3 & & \\
\hline ENETS stage & & & $<0.001$ & $1.586(1.505-1.673)$ \\
\hline I & 378 & 17.1 & & \\
\hline IIA & 250 & 11.3 & & \\
\hline IIB & 159 & 7.2 & & \\
\hline IIIA & 239 & 10.8 & & \\
\hline IIIB & 426 & 19.3 & & \\
\hline IV & 757 & 34.3 & & \\
\hline mENETS stage & & & $<0.001$ & $1.610(1.524-1.701)$ \\
\hline IA & 378 & 17.1 & & \\
\hline IB & 250 & 11.3 & & \\
\hline IIA & 159 & 7.2 & & \\
\hline IIB & 158 & 7.2 & & \\
\hline III & 507 & 23.0 & & \\
\hline IV & 757 & 34.3 & & \\
\hline rENETS stage & & & $<0.001$ & $1.612(1.525-1.703)$ \\
\hline IA & 378 & 17.1 & & \\
\hline IB & 250 & 11.3 & & \\
\hline IIA & 159 & 7.2 & & \\
\hline IIB & 141 & 6.4 & & \\
\hline III & 524 & 23.7 & & \\
\hline IV & 757 & 34.3 & & \\
\hline
\end{tabular}



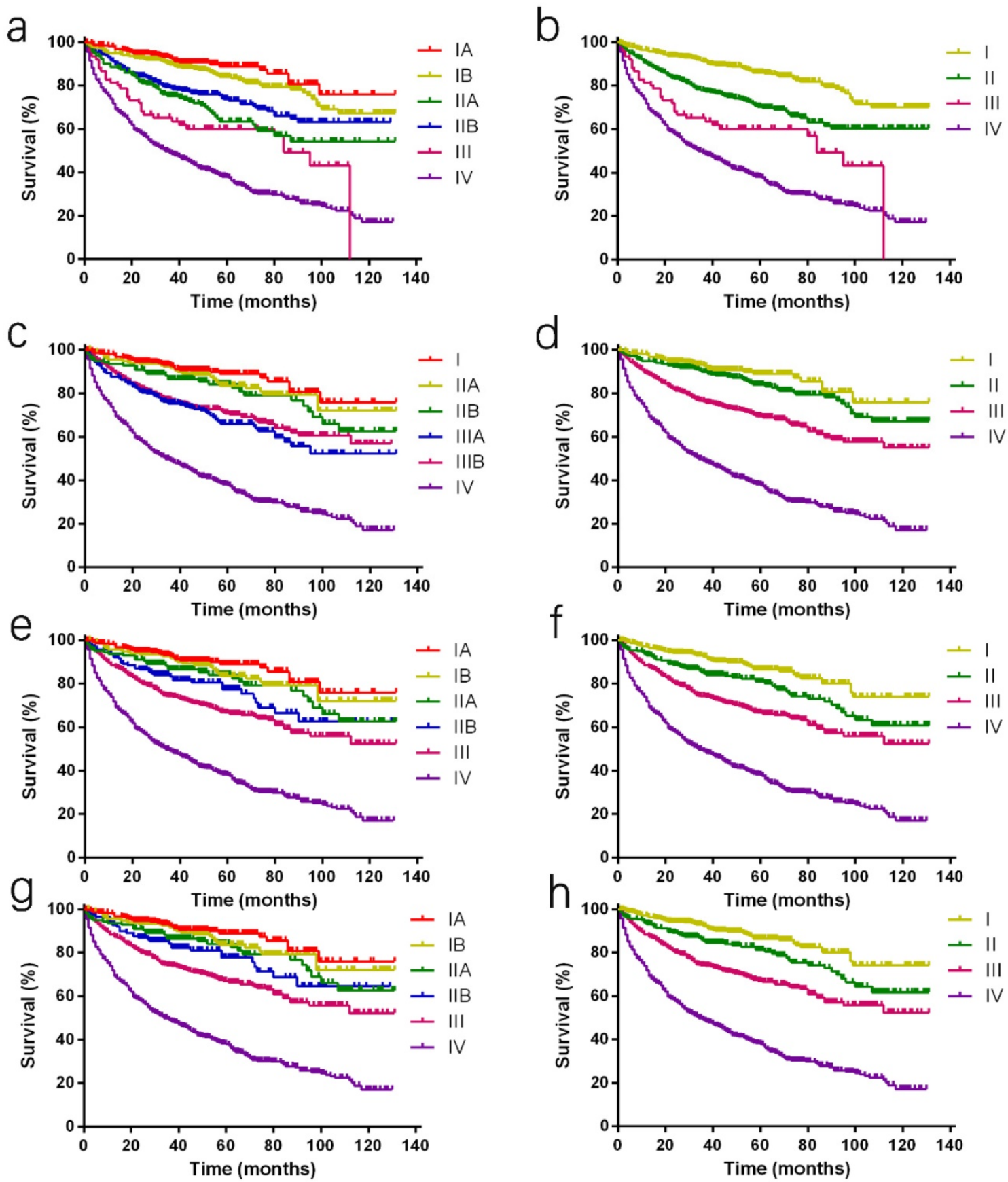

Figure 1. Kaplan-Meier curves of different staging classifications for patients from the SEER database. (a and b) AJCC 7th staging system; (c and d) ENETS; (e and f $)$ mENETS; and ( $g$ and $h$ ) rENETS staging system. Overlap existed between (a) the AJCC 7th classification stages III and IV disease and (c) the ENETS classification stages I and II disease. Survival curves were well separated by stage using $(\mathrm{e}, \mathrm{g})$ the modified ENETS classification and reformed ENETS classification. SEER: Surveillance, Epidemiology, and End Results; AJCC: American Joint Committee on Cancer; ENETS: European Neuroendocrine Tumor Society; mENETS: modified European Neuroendocrine Tumor Society; rENETS: reformed European Neuroendocrine Tumor Society.

\section{Modified ENETS staging system and survival}

Considering the above shortcomings of the AJCC 7th and ENET staging systems, mENETS staging classification was proposed previously by maintaining the ENETS T, N, and $\mathrm{M}$ definitions and adopting the AJCC 7th staging definitions. Through these changes, the proportion of patients of stage III disease using the mENETS staging classification was higher than that of the AJCC 7th staging classification $(23.0 \%$ v $3.6 \%$; Table 1). According to the mENETS classification, survival curves of all stages were well separated, which was further confirmed by the reasonable HRs with statistical significance (Fig. 1e and 1f; Table 2). 
Table 2. Multivariate analysis of prognostic factors.

\begin{tabular}{|c|c|c|c|c|c|c|c|c|}
\hline \multirow[t]{2}{*}{ Variables } & \multicolumn{2}{|c|}{ AJCC 7th stage } & \multicolumn{2}{|c|}{ ENETS stage } & \multicolumn{2}{|c|}{ mENETS stage } & \multicolumn{2}{|c|}{ rENETS stage } \\
\hline & $P$ value & HR $(95 \%$ CI) & $P$ value & HR $(95 \%$ CI) & $P$ value & HR $(95 \% \mathrm{CI})$ & $P$ value & HR $(95 \%$ CI) \\
\hline \multicolumn{9}{|l|}{ Age, year } \\
\hline$<40$ & 1 & & 1 & & 1 & & 1 & \\
\hline $40-59$ & 0.036 & $1.469(1.025-2.105)$ & 0.033 & $1.478(1.032-2.118)$ & 0.037 & $1.466(1.023-2.102)$ & 0.038 & $1.465(1.022-2.101)$ \\
\hline $60-79$ & $<0.001$ & $2.525(1.776-3.592)$ & $<0.001$ & $2.528(1.777-3.595)$ & $<0.001$ & $2.496(1.755-3.551)$ & $<0.001$ & $2.494(1.753-3.548)$ \\
\hline$\geq 80$ & $<0.001$ & $4.326(2.909-6.432)$ & $<0.001$ & $4.319(2.904-6.422)$ & $<0.001$ & $4.257(2.863-6.330)$ & $<0.001$ & $4.257(2.863-6.330)$ \\
\hline \multicolumn{9}{|l|}{ Sex } \\
\hline Female & 1 & & 1 & & 1 & & 1 & \\
\hline Male & 0.002 & $1.251(1.086-1.441)$ & 0.002 & $1.251(1.087-1.441)$ & 0.002 & 1.256 (1.091-1.447) & 0.002 & $1.255(1.090-1.445)$ \\
\hline \multicolumn{9}{|l|}{ Tumor type } \\
\hline Functional & 1 & & 1 & & 1 & & 1 & \\
\hline Nonfunctional & 0.402 & $1.097(0.883-1.363)$ & 0.400 & $1.098(0.884-1.363)$ & 0.364 & $1.106(0.890-1.373)$ & 0.370 & $1.104(0.889-1.372)$ \\
\hline \multicolumn{9}{|l|}{ Grade } \\
\hline High & 1 & & 1 & & 1 & & 1 & \\
\hline Intermediate & 0.019 & $1.251(1.038-1.509)$ & 0.021 & $1.246(1.033-1.502)$ & 0.021 & 1.247 (1.034-1.503) & 0.020 & $1.249(1.036-1.506)$ \\
\hline Low & $<0.001$ & 3.567 (3.021-4.213) & $<0.001$ & $3.562(3.017-4.206)$ & $<0.001$ & $3.585(3.036-4.234)$ & $<0.001$ & $3.584(3.035-4.232)$ \\
\hline \multicolumn{9}{|l|}{ Surgery } \\
\hline Performed & 1 & & 1 & & 1 & & 1 & \\
\hline $\begin{array}{l}\text { Recommended but not } \\
\text { performed }\end{array}$ & $<0.001$ & 3.289 (2.263-4.779) & $<0.001$ & $3.265(2.246-4.745)$ & $<0.001$ & $3.144(2.164-4.567)$ & $<0.001$ & $3.151(2.169-4.578)$ \\
\hline Not recommended & $<0.001$ & $2.765(2.325-3.289)$ & $<0.001$ & $2.783(2.348-3.300)$ & $<0.001$ & $2.752(2.322-3.260)$ & $<0.001$ & $2.749(2.320-3.257)$ \\
\hline \multicolumn{9}{|l|}{ Stage } \\
\hline I & 1 & & 1 & & 1 & & 1 & \\
\hline II & $<0.001$ & $1.988(1.556-2.540)$ & 0.154 & $1.338(0.897-1.995)$ & 0.001 & 1.735 (1.251-2.406) & 0.002 & $1.703(1.220-2.377)$ \\
\hline III & $<0.001$ & $2.236(1.527-3.274)$ & $<0.001$ & $2.429(1.706-3.459)$ & $<0.001$ & $2.270(1.718-3.998)$ & $<0.001$ & $2.271(1.722-2.996)$ \\
\hline IV & $<0.001$ & $3.194(2.531-4.031)$ & $<0.001$ & $3.813(2.680-5.423)$ & $<0.001$ & $3.537(2.707-4.622)$ & $<0.001$ & $3.540(2.709-4.625)$ \\
\hline
\end{tabular}

AJCC, American Joint Committee on Cancer; ENETS, European Neuroendocrine Tumor Society; HR, hazard ratio; mENETS, modified European Neuroendocrine Tumor Society; rENETS, reformed European Neuroendocrine Tumor Society.

\section{Reformed ENETS staging system and survival}

Compared to 7 th staging system, the rENETS staging system changed the $\mathrm{T}$ and $\mathrm{N}$ classification. The $\mathrm{N}$ classification was defined more precisely. The distinction between stage IIB and III disease using the rENETS staging classification was larger than that of the mENETS staging classification, so did stage II and stage III disease (Fig. $\mathbf{1 g}$ and $\mathbf{1 h}$ ). According to the rENETS staging system, survival curves were also separated as better as that in the mENETS staging system between stages and a tiny increase in HRs was observed with statistical significance (Table 2).

\section{Prognostic nomogram for OS}

One prognostic nomogram integrated the prognostic TNM staging system and all significant independent prognostic factors for OS was developed by multivariate Cox regression models. The C-index for OS prediction with the formulated nomogram containing rENETS staging system was 0.821 (95\% CI, 0.820 to 0.822 ), which was the same as the nomogram containing the mENETS staging system and higher than the C-index by other independent factors (Table 3). The higher $C$-index reflected the better predictive accuracy for OS achieved. Through the analysis of survival curves, HRs and C-index, the rENETS staging system showed the superior performance in the prediction for OS of pNETs, and nomogram containing rENETS staging system was formulated to predict the survival with larger advantage (Fig. 2).

\section{Validation of predictive accuracy of the nomogram with calibrations and decision curve analysis}

The calibration plot for the probability of OS at $5,8,10$ years showed optimal consistency between the observed OS and nomogram-predicted OS (Fig. 3a-c). The C-index of the formulated nomogram for OS prediction was 0.821 higher than that of the rENETS staging system or grade alone (0.710 and 0.689, respectively; Table 3).

Table 3. Discriminatory capabilities of nomogram and independent prognostic factors.

\begin{tabular}{lll}
\hline Variables & OS \\
\cline { 2 - 3 } & C-index & $\mathbf{9 5 \%}$ CI \\
\hline Nomogram (containing rENETS stage) & 0.821 & $0.820-0.822$ \\
Nomogram (containing mENETS stage) & 0.821 & $0.820-0.822$ \\
rENETS stage & 0.710 & $0.771-0.773$ \\
mENETS stage & 0.710 & $0.771-0.773$ \\
Grade & 0.689 & $0.688-0.670$ \\
\hline
\end{tabular}

rENETS, reformed European Neuroendocrine Tumor Society; mENETS, modified European Neuroendocrine Tumor Society; OS, overall survival.

On DCA, an original evaluation method to highlight predictive models with the clinical net benefit 22, 23, the formulated nomogram yielded superior net benefit along with a wider scope of threshold probability in contrast to the rENETS staging system alone (Fig. $\mathbf{3 d}$ and $\mathbf{3 f}$ ). This indicated higher predictive power for predicting 5-, 8-, 10-year OS. Meanwhile, higher threshold probability levels meant superior evaluation of decision outcomes. 


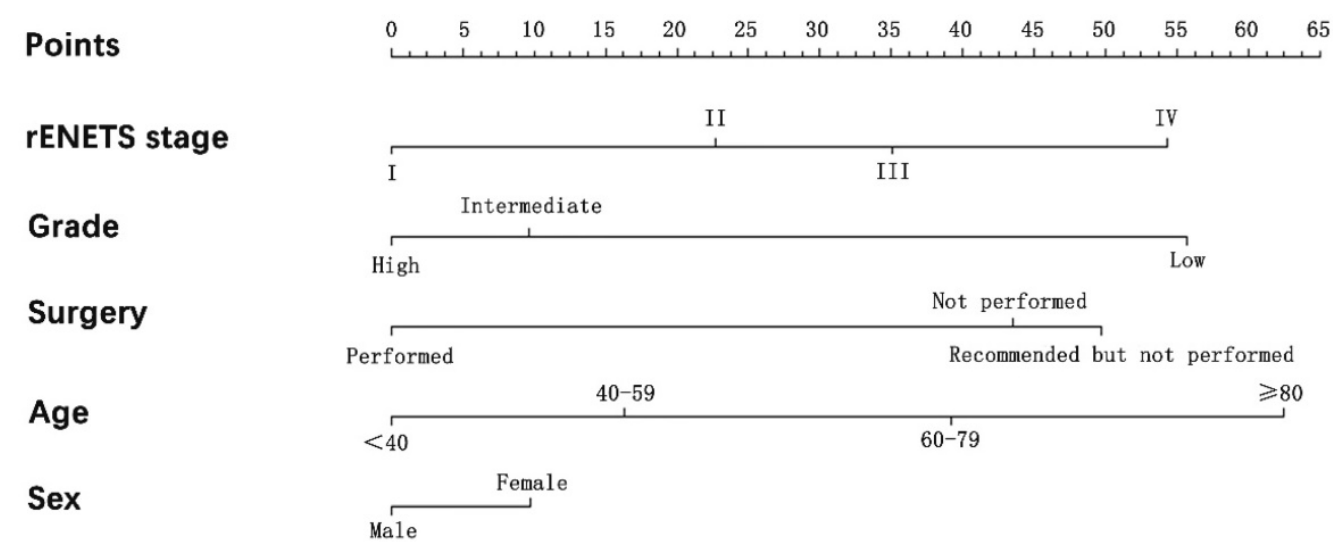

\begin{tabular}{llllllllllllll}
\hline Total Points & 0 & 20 & 40 & 60 & 80 & 100 & 120 & 140 & 160 & 180 & 200 & 220 & 240
\end{tabular}

Linear Predictor

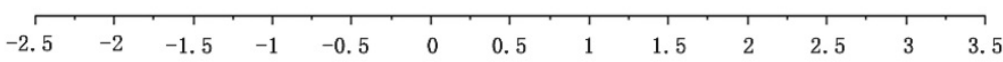

5-year OS

\begin{tabular}{llllllllll}
\hline 0.9 & 0.8 & 0.7 & 0.6 & 0.5 & 0.4 & 0.3 & 0.2 & 0.1
\end{tabular}

8-year OS

10-year OS

$\begin{array}{lllllllll}0.9 & 0.8 & 0.7 & 0.6 & 0.5 & 0.4 & 0.3 & 0.2 & 0.1\end{array}$

$\begin{array}{llllllllllll}0.9 & 0.8 & 0.7 & 0.6 & 0.5 & 0.4 & 0.3 & 0.2 & 0.1\end{array}$

Figure 2. Prognostic nomogram analysis for pNETS. The nomogram predicts OS in patients with pNETs (to use the nomogram, an individual patient's value is located on each variable axis, and a line is drawn upwards to determine the number of points received for each variable value. The sum of these number is located on the total points axis, and a line is drawn downwards to the survival axes to determine the likelihood of 5-, 8-, 10-year OS). pNETS, pancreatic neuroendocrine tumors; OS, overall survival; rENETS, reformed European Neuroendocrine Tumor Society.

\section{Discussion}

In this study, over 2,000 patients from SEER database were systematically analyzed. Consistent to a previous study ${ }^{7}$, the lower proportion of stage III disease $(3.6 \%)$ with the AJCC 7th staging system, or a similar prognosis between patients with stage I disease and stage II disease $(P=0.154)$ using the ENETS staging system suggested that both of them were far from a rational classification for pNETs. In addition, the HR of death for patients with stage IIIB disease was also found lower than that for patients with stage IIIA disease in the ENETS staging system (HR for stage IIIA, 3.325; HR for stage IIIB, 3.053, with stage I as the reference). In the comparison between the mENETS and rENETS staging system, we could directly discover that the changes of HR for patients in stage II and III disease, or stage III and IV disease with the mENETS staging system was slightly lower than that with the rENETS staging system, so did the discrimination ability in the survival curves. In the univariate and multivariate analysis, rENETS staging system, grade, surgery, age and sex were finally discovered as the independent prognostic factors. One novel nomogram was established to predict the 5-, 8- and 10- OS rates of pNET patients based on all the independent prognostic factors. The C-index of the formulated nomogram was 0.821 which was larger than other risk factors alone. Furthermore, calibrations and DCA validated our nomogram. The large sample size and wide distribution of patients in this cohort could guarantee its representativeness for pNETs.

Controversy still remains on whether the patients' survival is related to lymph node metastasis ${ }^{24-27}$. Although Krampit et al. ${ }^{25}$ had considered the links between the number of lymph nodes and survival, he just applied two lymph nodes as the cut-off value, which was limited and blurry. One major character of pNETs was its tendency of metastasis and recurrence, and many studies confirmed that distant metastasis could promote nodal metastasis and unfavorably influenced the survival of pNET patients ${ }^{28-30}$. In our study, stage IV disease accounted for more than $30 \%$, showing the necessity of ideal nodal substage for pNTEs. The N classification in AJCC 8th staging system performed better in risk stratification of pancreatic ademocarcinoma ${ }^{20}$, so was the incorporation of AJCC 8th N-stage revision into the ENETS staging system performed superior prediction in survival of pNET patients. In a previous study, Luo et $\mathrm{al}^{31}$ had illustrated the significance of $\mathrm{N}$ classification in pNETs patients, however, one single study is unlikely to provide enough information to illustrate the efficacy of the chosen $\mathrm{N}$ classification, which even 

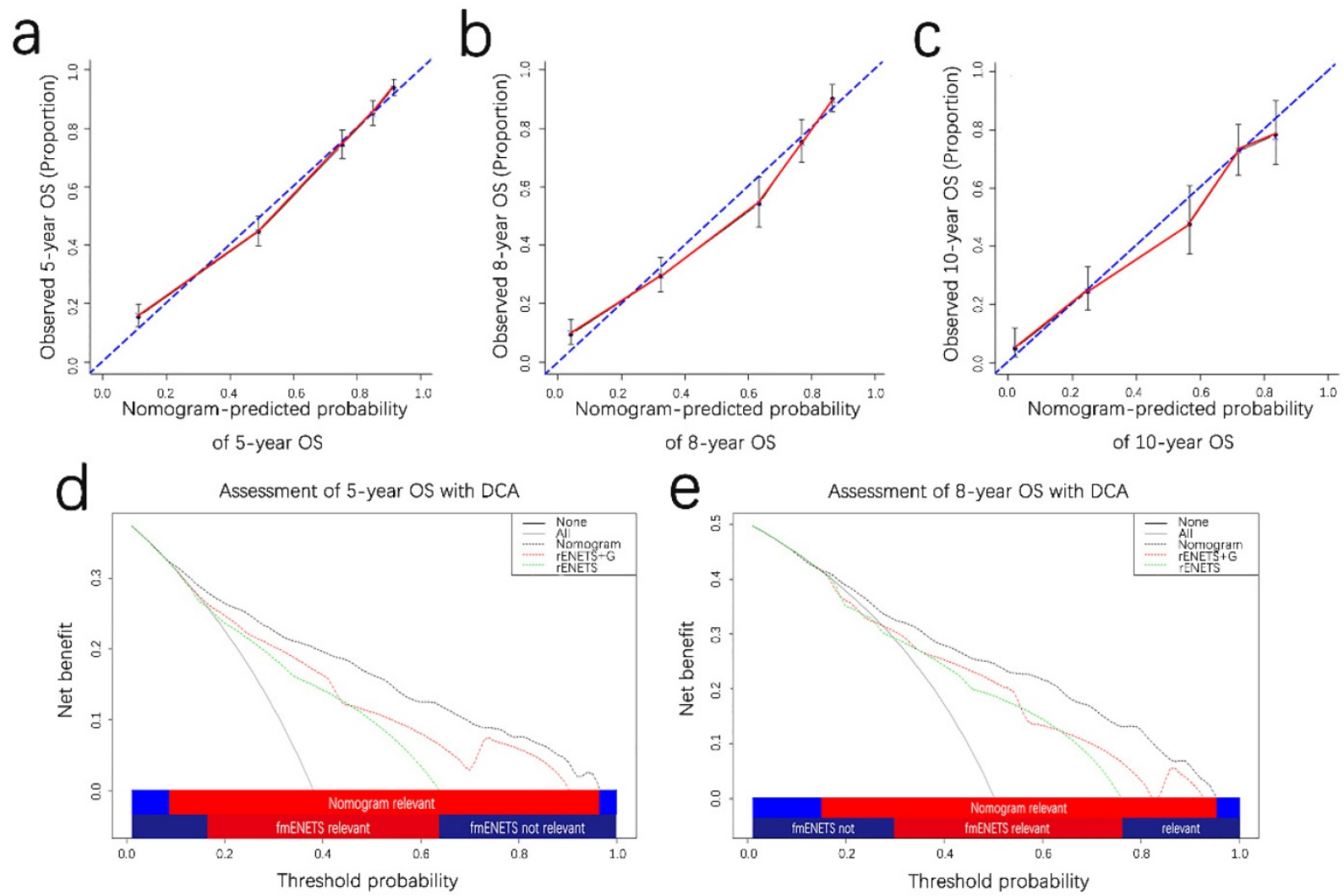

of 8 -year OS

of 10 -year OS
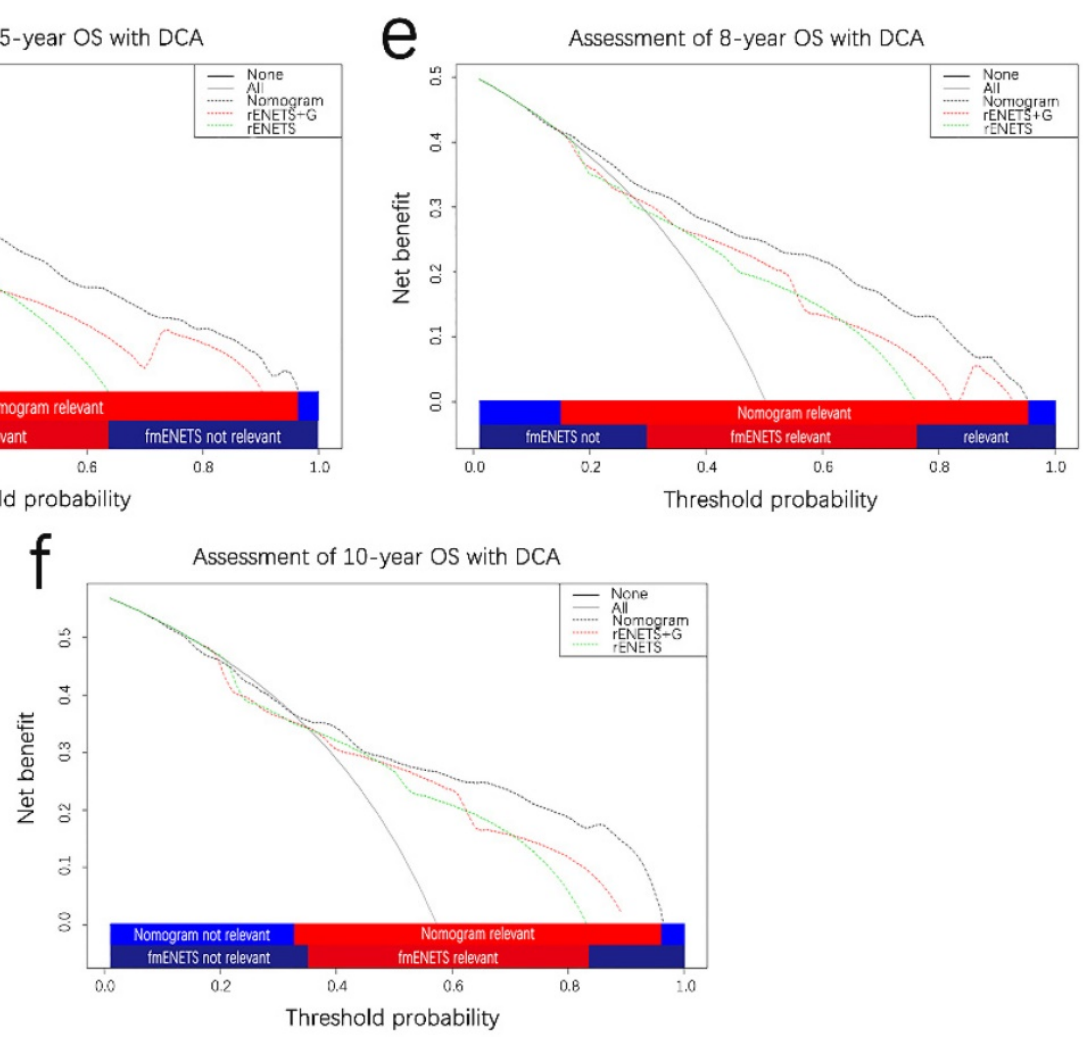

Figure 3. Prognostic calibration curves and decision curve analysis for pNETS. The calibration curves predict OS at 5 years (a), 8 years (b) and 10 years (c) in the patient with PNETs. Decision curve analyses depict the clinical net benefit in pairwise comparisons across the different models. Nomogram is compared with the rENETS staging system in terms of 5-(d), 8-(e), 10-year(f) OS in the patient with pNETs. Dashed lines indicate the net benefit of the predictive models across a range of threshold probabilities (black: nomogram; red: rENETS staging system+G; green: rENETS staging system. The horizontal solid black line represents the assumptions that no patient will experience the event, and the solid grey line represents the assumption that all patients will experience the event. On decision curve analysis, the nomograms showed superior net benefit compared with rENETS+G and rENETS staging system across a wider range of threshold probabilities. pNETS, pancreatic neuroendocrine tumors; OS, overall survival; rENETS, reformed European Neuroendocrine Tumor Society.

possibly could lead to selection bias. The AJCC 8th N classification was widely used in pancreatic tumor and confirmed its validity. A highlighted improvement was obtained with our revised rENETS staging system and more clinical decisions may benefit from it.

Except the TNM staging system, there are a plethora of other indicators for the prognosis of pNET patients. Through our analysis, the grade, surgery, age and sex were independent predictors for OS, consistent to the previous reports ${ }^{27,32}$. Grade reflected tumor biological behavior and was closely connected to its prognosis ${ }^{33}$. Lianyuan et al. ${ }^{34}$ had confirmed that surgical resection of primary lesion was associated with OS $(\mathrm{P}=0.025)$ benefit as well as other studies ${ }^{35}$. Non-resection was a potential risk factor associated with poor survival. From the constructed nomogram, we found the pNET patients who had tumor resected obtained better survival. Intriguingly, the patients who was recommended with surgery but not performed finally had higher risk than those was not recommended, which suggested reduction in tumor load was significant to survival of pNETs. The age, surgery, grade and TNM staging system were all 
attributed in high risk factors with wider range of points. However, sex as an independent predictor had relatively lower risk.

Validation of the formulated nomogram was significant to avoid overfitting of the model and reveal the representativeness ${ }^{36}$. In this study, calibration curves showed a superior consistency between nomogram-predicted and actually observed probability, and DCA revealed superior clinical benefit with a wider range of threshold probability, which could guarantee the reliability of the formulated nomogram. Its limitations were retrospective in nature and lack of blood serum indices and specific proteins, so prospective multicenter clinical trials should be carried out to make further efforts in validating our results and comparing to other models.

In conclusion, the novel rENETS staging system showed excellent performance in the risk stratification and the formulated nomogram could obtain a wider range in prognostic prediction for pNETs.

\section{Abbreviations}

mENETS: modified European Neuroendocrine Tumor Society; pNETs: pancreatic neuroendocrine tumors; AJCC: American Joint Committee on Cancer; ENETS: European Neuroendocrine Tumor Society; rENETS: reformed European Neuroendocrine Tumor Society; PDAC: pancreatic ductal adenocarcinoma; SEER: Surveillance, Epidemiology, and End Results; CS: collaborative stage; HR: hazard ratios; OS: overall survival; C-index: concordance index; DCA: decision curve analysis.

\section{Acknowledgements}

This work was supported by the National Natural Science Foundation of China (No. 81401923, 81572294, 81702304, 81773068 and 81702964) and Shanghai Municipal Commission of Health and Family Planning (No. 20160277).

\section{Informed consent}

The primary and processed data were downloaded from SEER database. All SEER data were available with no restriction in presentations or publications according to its guidelines.

\section{Competing Interests}

The authors have declared that no competing interest exists.

\section{References}

1. Yamamoto $Y$, Okamura $Y$, Uemura S, et al. Vascularity and Tumor Size are Significant Predictors for Recurrence after Resection of a Pancreatic Neuroendocrine Tumor. Ann Surg Oncol. 2017;24:2363-70.
2. Halfdanarson TR, Rabe KG, Rubin J, et al. Pancreatic neuroendocrine tumors (PNETs): incidence, prognosis and recent trend toward improved survival. Ann Oncol. 2008;19:1727-33.

3. Kim SJ, An S, Lee JH, et al. Loss of Progesterone Receptor Expression Is an Early Tumorigenesis Event Associated with Tumor Progression and Shorter Survival in Pancreatic Neuroendocrine Tumor Patients. J Pathol Transl Med. 2017;51:388-95.

4. Lee YS, Kim H, Kim HW, et al. High Expression of MicroRNA-196a Indicates Poor Prognosis in Resected Pancreatic Neuroendocrine Tumor. Medicine (Baltimore). 2015;94:e2224.

5. Yao JC, Hassan M, Phan A, et al. One hundred years after "carcinoid": epidemiology of and prognostic factors for neuroendocrine tumors in 35,825 cases in the United States. J Clin Oncol. 2008;26:3063-72.

6. Canellas R, Lo G, Bhowmik S, et al. Pancreatic neuroendocrine tumor: Correlations between MRI features, tumor biology, and clinical outcome after surgery. J Magn Reson Imaging. 2017.

7. Luo G, Javed A, Strosberg JR, et al. Modified Staging Classification for Pancreatic Neuroendocrine Tumors on the Basis of the American Joint Committee on Cancer and European Neuroendocrine Tumor Society Systems. J Clin Oncol. 2017;35:274-80.

8. Murphy CE, McCormick KA, Shankaran V, et al. Grade Assignment by Ki-67 Proliferative Index, Mitotic Count, and Phosphohistone H3 Count in Surgically Resected Gastrointestinal and Pancreatic Neuroendocrine Tumors. Pancreas. 2017;46:1359-65.

9. Bilimoria KY, Bentrem DJ, Merkow RP, et al. Application of the pancreatic adenocarcinoma staging system to pancreatic neuroendocrine tumors. J Am Coll Surg. 2007;205:558-63.

10. Kloppel G, Rindi G, Perren A, et al. The ENETS and AJCC/UICC TNM classifications of the neuroendocrine tumors of the gastrointestinal tract and the pancreas: a statement. Virchows Arch. 2010;456:595-7.

11. Rindi G, Kloppel G, Alhman H, et al. TNM staging of foregut (neuro)endocrine tumors: a consensus proposal including a grading system. Virchows Arch. 2006;449:395-401.

12. Jensen RT, Cadiot G, Brandi ML, et al. ENETS Consensus Guidelines for the management of patients with digestive neuroendocrine neoplasms: functional pancreatic endocrine tumor syndromes. Neuroendocrinology. 2012;95:98-119.

13. Halfdanarson TR, Bamlet WR, McWilliams RR, et al. Risk factors for pancreatic neuroendocrine tumors: a clinic-based case-control study. Pancreas. 2014;43:1219-22.

14. Rindi G, Falconi M, Klersy C, et al. TNM staging of neoplasms of the endocrine pancreas: results from a large international cohort study. J Natl Cancer Inst. 2012;104:764-77.

15. Han $X$, Zhang $C$, Tang $M$, et al. The value of serum chromogranin $A$ as a predictor of tumor burden, therapeutic response, and nomogram-based survival in well-moderate nonfunctional pancreatic neuroendocrine tumors with liver metastases. Eur J Gastroenterol Hepatol. 2015;27:527-35.

16. Ferrara F, Luigiano C, Maimone A, et al. Simultaneous EUS-FNA Diagnosis and TNM Staging of a Pancreatic Neuroendocrine Tumor in a Patient with an Unrecognized MEN Type 1. Case Rep Oncol Med. 2012;2012:619428.

17. Yang M, Zeng L, Zhang Y, et al. TNM staging of pancreatic neuroendocrine tumors: an observational analysis and comparison by both AJCC and ENETS systems from 1 single institution. Medicine (Baltimore). 2015;94:e660.

18. Liu TC, Hamilton N, Hawkins W, et al. Comparison of WHO Classifications (2004, 2010), the Hochwald grading system, and AJCC and ENETS staging systems in predicting prognosis in locoregional well-differentiated pancreatic neuroendocrine tumors. Am J Surg Pathol. 2013;37:853-9.

19. Strosberg JR, Cheema A, Weber JM, et al. Relapse-free survival in patients with nonmetastatic, surgically resected pancreatic neuroendocrine tumors: an analysis of the AJCC and ENETS staging classifications. Ann Surg. 2012;256:321-5.

20. Kamarajah SK, Burns WR, Frankel TL, et al. Validation of the American Joint Commission on Cancer (AJCC) 8th Edition Staging System for Patients with Pancreatic Adenocarcinoma: A Surveillance, Epidemiology and End Results (SEER) Analysis. Ann Surg Oncol. 2017;24:2023-30.

21. $\mathrm{Pu} \mathrm{N}, \mathrm{Lv}$ Y, Zhao G, et al. Survival prediction in pancreatic cancer patients with no distant metastasis: a large-scale population-based estimate. Future Oncol. 2018;14:165-75.

22. Vickers AJ, Elkin EB. Decision curve analysis: a novel method for evaluating prediction models. Med Decis Making. 2006;26:565-74.

23. $\mathrm{Pu} \mathrm{N}, \mathrm{Li} \mathrm{J}, \mathrm{Xu} \mathrm{Y}$, et al. Comparison of prognostic prediction between nomogram based on lymph node ratio and AJCC 8th staging system for patients with resected pancreatic head carcinoma: a SEER analysis. Cancer Manag Res. 2018;10:227-38.

24. Taki K, Hashimoto D, Nakagawa S, et al. Significance of lymph node metastasis in pancreatic neuroendocrine tumor. Surg Today. 2017:47:1104-10.

25. Krampitz GW, Norton JA, Poultsides GA, et al. Lymph nodes and survival in pancreatic neuroendocrine tumors. Arch Surg. 2012;147:820-7.

26. Ferrone CR. Lymphadenectomy for pancreatic neuroendocrine tumors: is that the relevant debate? Ann Surg. 2014;259:213-4.

27. Liu $\mathrm{P}$, Zhang $\mathrm{X}$, Shang $\mathrm{Y}$, et al. Lymph node ratio, but not the total number of examined lymph nodes or lymph node metastasis, is a predictor of overall survival for pancreatic neuroendocrine neoplasms after surgical resection. Oncotarget. 2017.

28. Tao M, Yuan C, Xiu D, et al. Analysis of risk factors affecting the prognosis of pancreatic neuroendocrine tumors. Chin Med J (Engl). 2014;127:2924-8. 


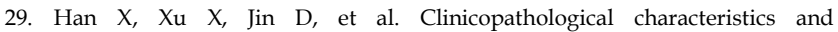
prognosis-related factors of resectable pancreatic neuroendocrine tumors: a retrospective study of 104 cases in a single Chinese center. Pancreas. 2014;43:526-31.

30. Parekh JR, Wang SC, Bergsland EK, et al. Lymph node sampling rates and predictors of nodal metastasis in pancreatic neuroendocrine tumor resections: the UCSF experience with 149 patients. Pancreas. 2012;41:840-4.

31. Luo G, Jin $\mathrm{K}$, Cheng $\mathrm{H}$, et al. Revised nodal stage for pancreatic neuroendocrine tumors. Pancreatology. 2017;17:599-604.

32. Conrad C, Kutlu OC, Dasari A, et al. Prognostic Value of Lymph Node Status and Extent of Lymphadenectomy in Pancreatic Neuroendocrine Tumors Confined To and Extending Beyond the Pancreas. J Gastrointest Surg. 2016;20:1966-74.

33. Raj N, Valentino E, Capanu M, et al. Treatment Response and Outcomes of Grade 3 Pancreatic Neuroendocrine Neoplasms Based on Morphology: Well Differentiated Versus Poorly Differentiated. Pancreas. 2017;46:296-301.

34. Lianyuan T, Dianrong $X$, Sadula A, et al. Surgical resection of primary tumor improves survival of pancreatic neuroendocrine tumor with liver metastases. Oncotarget. 2017.

35. Zhang JW, Che X, Lan ZM, et al. [Surgical treatment and prognosis of pancreatic neuroendocrine carcinoma]. Zhonghua Zhong Liu Za Zhi. 2016;38:925-8.

36. Iasonos A, Schrag D, Raj GV, et al. How to build and interpret a nomogram for cancer prognosis. J Clin Oncol. 2008;26:1364-70. 